\title{
Abdominal Aortic ANEURYSM Identification Using HLSFMM Segmentation and SVM Classifier
}

\author{
S. Anandh, R. Vasuki, Raid Al Baradie
}

\begin{abstract}
The localized inflammation of the abdominal aorta region causes Abdominal Aortic Aneurysm (AAA). The width of the lumen enlarges its size $3 \mathrm{~cm}$ or more than half of its diameter, which is larger than the typical diameter. There is no symptom until it becomes ruptured, which may often results in death. In this paper, a hybrid level set technique is presented to detect and segment the image taken from MRI of abdominal aortic aneurysm region. In traditional level set technique re-initialization problems are high. This problem is completely eradicated in the Hybrid Level Set Fast Marching method (HLSFMM). Median filter diminishes the noise in the image efficiently when compared to standard SVM classifier which uses Gaussian RBF kernel operator as a diameter measure by incorporating spatial data. Finally HLSFMM is utilized to extract source boundary in pre segmentation stage. The precision and the orderliness of the proposed method are extracted for different noisy MRI AAA images. Compared this result with other methods, the proposed system is much proficient for images with noises and accurate segmentations results are attained.
\end{abstract}

Keywords : Abdominal Aortic Aneurysm, Median filter, HLSFMM segmentation, SVM Classifier.

\section{INTRODUCTION}

Abdominal Aortic Aneurysm (AAA) is a vascular ailment arises due to degenerative, provocative, mycotic ailments and additionally by arteriosclerosis. It results in broadening of the stomach aorta because of debilitated aortic divider. At whatever point left untreated AAA will enlarge following a number of occasions expanding the danger of aortic divider happiness [1]. AAA impacts around $0.2 \%$ of individuals after 65 years of age. Half of AAA is distinguished by shot and for nearly $0.9 \%$ of the AAA affected patients will be cracked because of debilitated blood vessel dividers. As a deadly outcome, $70-90 \%$ of patients with cracked AAA will no more exist [2]. The different choices of treatment available today, however intrusive method is recommended in light of endovascular situation of aortic stent joins through an

Revised Manuscript Received on December 30, 2019.

* Correspondence Author

S. Anandh*, department of Biomedical Engineering, Bharath University, Chennai (Tamil nadu) Inida. E-mail: anandhcsiit@gmail.com

Dr. R. Vasuki, department of Biomedical Engineering, Bharath University, Chennai (Tamil nadu) Inida. E-mail: rkaran02@gmail.com

Dr. Raid Al Baradie, department of Medical Lab, Majmaah University, Kingdom of Saudi Arabia. E-mail: r.albaradie@mu.edu.sa

(C) The Authors. Published by Blue Eyes Intelligence Engineering and Sciences Publication (BEIESP). This is an open access article under the CC BY-NC-ND license (http://creativecommons.org/licenses/by-nc-nd/4.0/) insignificantly obtrusive space on the patient's body. So as to play out this methodology effectively, the fitting stent join gadget must be chosen. Keeping in mind the end goal to pick the stent unite of proper structure and size, exact data on aortic size and measure is needed. Present day therapeutic image processing strategies pursued by fitting picture investigation techniques [3] have appeared to be valuable for estimations of AAA [4]. Cardiovascular infection, specifically aortic aneurysm (AA), is the thirteenth driving reason for the loss of life in US [6]. There are a few treatment choices available for aortic aneurysms. One alternative is open medical procedure in which the body of patient is unlatched at the aneurysm area and a stent is screwed in the vascular prosthesis tissue. This type of intercession is exceptionally unpleasant to the patient and not suits for everybody. An elective choice is endovascular stent arrangement. Addition of this stent is finished by a catheter method for the most part it via a little slit in the femoral course $[7,8]$. The aneurysm breadth is the primary essential pointer deciding if an intercession should happen. If there should be an occurrence of a stomach aortic aneurysm (AA), move is made if the measurement is symmetrical with the vessel centreline is more than 55 millimeter. In this, a few techniques are proposed to help clinician regarding the division of AA. A self-loader isoperimetric calculation for AA division is utilized [9].

A functioning appearance demonstrates has likewise been utilized for AA division [10]. Lu et al acquainted a methodology with portion the internal and external shape of thoracic aortic aneurysm (TAA) with a functioning form demonstrating an imperative to keep the shape at a specific separation [11]. Bruijne et al exhibited a technique to fragment tubular structure in light of dynamic visual model and have been connected it to AAA division [12]. Heiman et al. depict a strategy that unequivocally distinguishes a division spill by figuring a way between two client characterized focuses one inside the division spill district and another within the objective locale. This needs client association, is restricted to a solitary bottleneck shaped spill and can't deal with holes to a few neighboring structures [13]. Distinctive answers for the aorta division are seen in inscription. The incorporated vessel hub extrication and fringe calculation, neural systems, area developing or watershed and level set dependant methodologies [14]. Behrens depicted a Hough change based calculation joined with a kalman channel. 
The disadvantage of this methodology was, in any case, the requirement for three clients chose parameters in particular, the beginning stage of the aorta, the aorta span, and a rough hub bearing. He built up a completely programmed division strategy for the aorta, utilizing a Hough change based deformable surface model invention. In contrast with Behrens method, the proposed technique joins progressively from the earlier anatomical learning regarding the state of the aortic curve and subsequently no manual instatement is required [15]. In order to leveling the CTA images, an anti geometric dispersion method was used. The Lumens are found from the pre-processed image by two steps first segmentation then morphological operation. Confinement of the abdominal lumens is established by applying geometric data, scientific morphology and clustering experimentation. It is very difficult to segment the inner and outer wall of the AAA region. Hence the systematic segmentation and measurement of AAA is done in [16]. To identify the thrombus in the cardiovascular system, 3-D level set and anisotropic dispersal are used and 3D CTA image data is applied here. The geometric deformable model such as Active contours methods depending on region or edge by utilizing partial distinctive equation (PDE) executed by level sets. The Courant Friedrichs Lewy (CFL) is a stability condition for explicit Finite Distinctive Method (FDM) for tracking curves. For delineating and reducing the connections at fragile boundary, a diffusion function has been used [17]. For key measurements of the necessitate stent candidate has been considered for appropriate, measurements from the images. Automatic detection and segmentation is realized from 3D attribute of the CT. First luminal centre is identified for segmentation. The measurements of diameter are in vertical with the vessel direction and a real 3D path length of the lumens is followed by ESM for each matched region [18]. Using RF data the B-mode images were reconstructed and further image processing was performed in Mathematics. For the image filtering process Laplacian of Gaussian (LoG) filter is used which boosts the vessel wall. On the other hand, the angle may be inaccurate and also the placement of the ultrasound transducer is a physical process [19]. To produce FE mesh for the systematic analysis of AAA from the standard medical CTA data, a hierarchical method is developed. Discretization is denoted by a hexahedral-dominated mesh. Users without skills can be handles' this method in medical image processing and computational mechanics [20].

In the present system introduces a new method called hybrid level set for increasing the efficient segmentation, accurate boundary values with curves are obtained. Compared with the conventional level set methods in hybrid level set model eliminates the reinitialization problems. It is implemented in some areas like medical image processing, remote sensing and in computerized vision. In noisy images the drawback is reduced and computation time is reduced using the median filter and spatial kernel fuzzy c means clustering. First the noise is reduced by applying median filter then spatial kernel fuzzy c means clustering is used in order to get the de noised image. The final step is applying hybrid level set to extract the object boundary using pre-segmentation procedure. The precision and the orderliness of the proposed method for various abdominal aortic images are described. Hence the experiment shows that it is very efficient method for the noisy images and accurate segmentation is applied.

\section{RECENT WORKS}

Several existing approaches which were described previously for change detection issues are given below:

Moti Freiman et al. [21], present an iterative model-compelled diagram cut calculation to divide Abdominal Aortic Aneurysm (AAA) thrombus. Provided an underlying division of the aortic lumens, the proposed technique consequently portions the thrombus by repeatedly integrated force dependent diagram min-split division and geometrical parametric models fitting. The geometric models successfully compel the chart min-cut division from "spilling" to close-by vein and organ. Exploratory outcomes on AAA CT dataset yields hearty divisions of the AAA thrombus quickly PC time with an average outright size contrast of $10.0 \%$ and average volume cover mistake of $11.9 \%$ with practically identical to the bury onlooker blunder. Roger C. Cap et al. [22] built up the new procedure as volume rendering. It is a significant and essential system for logical perception. One surely understood application region is the reproduction and representation of yield from medicinal scanner like figured tomography. 2-D grey value cuts created by the scanner are reproduced and shown as a 3D display. Size perception of restorative pictures should find two critical issues. In the primary place, it is hard to portion restorative outputs as individual material in light of on force esteems. This would results in volume which contains a lot of irrelevant or superfluous materials. Next, despite the fact that greyscale pictures are the ordinary technique for showing therapeutic volumes; these sorts of pictures are not really fitting for featuring areas of enthusiasm inside the volume. Investigations of the practical framework have demonstrated that each of the power esteem is hard to identify in a greyscale picture. In such circumstances, shading is much viable visual element, as the low stage visual framework could quickly and precisely identify the nearness or nonappearance of a specific target shading in a multiple hued picture. Achia Kronman et al. [23] present another technique for the location and adjustment of division spills in volumetric medicinal pictures. Division spills happen if the division volume grows external to the real target limit as a neighbouring structure. This technique distinguishes the division spill premise limit by angle size probability characterization, fits a surface up to the break premise limit, and isolates the hole from the objective structures in the wake of finding their basic limit. Our strategy is programmed, does not depend on earlier data, and is free of the underlying division technique. Exploratory assessment on 140 divisions of kidney and stomach aortic aneurysm from tomography filters using basic division strategies yield a change of 55.6\% $(s t d=14.5)$ and $49.5 \%(s t d=19.4)$ in the average surface separation and the volume cover blunder among the underlying and the remedied divisions. Egger J et al. [24] presented a technique for sectioning the epithelial (external) level of aoric aneurysm in tomography-angiography acquisition is exhibited. Our technique begins by registering the lumens centreline among the clients characterized seed focuses which fill in as limits to the degree of the injury. 
The centreline decides the Multiple Planar Reconstruction (MPR) plane which shows the vessel hybrid segments. At that point, the client physically sections the epithelial surface of the primary proximal MPR planes to resize a form. Starting here, a circle is drawn: Initially, the shape is utilized to form a confined pursuit locale in the neighbouring distance MPR planes via the centreline. The voxel of the adjoining MPR planes inside this pursuit district and the voxel on which the shape itself suits are utilized to make a coordinated diagram. At that point, the mincut of the diagram is computed, making an ideal division of the AA verge on the nearby MPR planes. This fragmented shape fills in as a beginning stage for the circle, which is then executed over again.

Josu Maiora et al. [25], show a technique for AAA. It is a neighbourhood enlargement of the Aorta which happens among the renal and iliac corridors. The debilitating of the aortic divider prompts its distortion and generates thrombus. As of late, this method utilizes treatments which include the addition of an endovascular prosthetic (EVAR), the benefit of which is insignificant intrusive methodology, yet additionally expects observing to break down postoperative patient results. So as to adequately survey the progressions experienced after medical procedure, it is important to section the aneurysm, which is an exceptionally tedious assignment. Here we depict the underlying consequences of a new dynamic learning mixture scheme for the self-loader identification and division of the lumens and the AAA thrombus, which utilizes picture force highlights and discriminative Random Forest classifiers.

Stefanie et al. [26], proposed a crossbreed deformable modeling technique for segmenting the AAA thrombus using NURBS surface which functions on two parameters mapping to a surfaces in 3-D space. B-Spline surface is used to design the deformation therefore; it gives stronger smoothness constraints non-uniform distribution. Data sets are big in size; so better performance is reached by this deformation modeling. A less estimation is needed because of the less amounts of operating points. NURBS surface smoothness limitations of the division avoid leakage in the nearby region. Most accurate results are obtained by this algorithm. The minor manual post processing is acceptable which is visually inspected by the medical experts. But the segmentation result accuracy is not obtained till now. Splitting and merging of structures of our deformation model should be allowed. Difficult bifurcation position is a major drawback of this model.

Tuan D. Pham et al. [27] presents a new approach of a nonlinear dynamic modeling and stochastic structure categorization of AAA. Image markers are used for the determination and management of rupture risk treatment which requires no accurate AAA image division. Measuring the regularity of image signals is the main concept of this process. Nonlinear dynamic model combined with hidden Markov model is developed to remove imaging markers for danger prediction. Testing of more inclusive data is needed which is the main disadvantage of this method.

T. J. Ellis et al. [28] proposed another approach of structural segmentation from 3-D medical datasets. Partial Distinctive Equation (PDE) is used in Level sets and it is widely applied in image segmentation. Comparing to conventional level set methods, the velocity of curve development and the division results are enhanced considerably. The edge based method requires less parameter and the region dependent method requires more parameter and setting of these parameters is more complicated due to complex structure. In this paper, the intensity issue in homogeneities and the fragile boundaries in the images are reduced. But division of the thrombus size is challenging hence accurate results cannot be obtained.

Cosmin et al [29]. Proposed a method for segmentation uses multiplanar reformatted slice (MPR). In 3-D CT information, thrombus cannot be easily identified from neighboring tissues which are external to the aorta. Machine learning techniques and extra attributes to detect the aortic outer walls are combined together, which contains both lumens and thrombus. The graph-based method intended for recovering the aortic boundaries in multiple planar reformatted slices, the channel responses of a phase congruency channel and distance dependent components are the cost modules of this technique. For this classifier, hyper parameters are found by using evolutionary algorithm. The separation of the true lumen and thrombus is the toughest task. The kNN has achieved better results compared to the SVM, but in rare cases it is a disadvantage of this method.

Marleen et al [30] presents a methodology for localizing and segmenting bifurcated aortic endograft using computerized tomography angiography (CTA) pictures. The user point out the primary and the final marker, and the rest of the markers are identified without human intervention. There are three stages, a graft contour detection is performed initially in the CT-slice, then the reformatted slices are aligned orthogonal with the approximate graft axis, and the segmentation is utilized from the next method which performs contour detection. The advantage of this method is, when the marker location is identified, its artifact can be detected. The method may be made more robust .The accuracy is less that less accurate diameter measurements, graft curvature and torsion measurements.

\section{PROPOSED SYSTEM DEVELOPMENT}

In this method partial differential equation by a numerical scheme is applied, Curve circulation is a famous method. The core idea is to create an initial curve in the direction of the minimum cost potential function. These methods are a conceptual framework for a tool for numerical analysis of surfaces and shapes. The essential process involved in the proposed segmentation algorithm is given in figure 1. A suitable filter is applied on the part of the AAA MRI dataset to obtain the ROI thereby reducing the segmentation time. For this, median filter is used to reduce the noise in the image second stage SKFCM is applied to extracts image finally HLSFMM is applied to segment the accurate boundary images. To segment the thrombus boundary, the HLSM is utilized initially to the thrombus for obtaining the boundary curves by which the thrombus boundary is reconstructed. More than one boundary is detected in level set methods simultaneously, and multiple initial contours can be placed. 


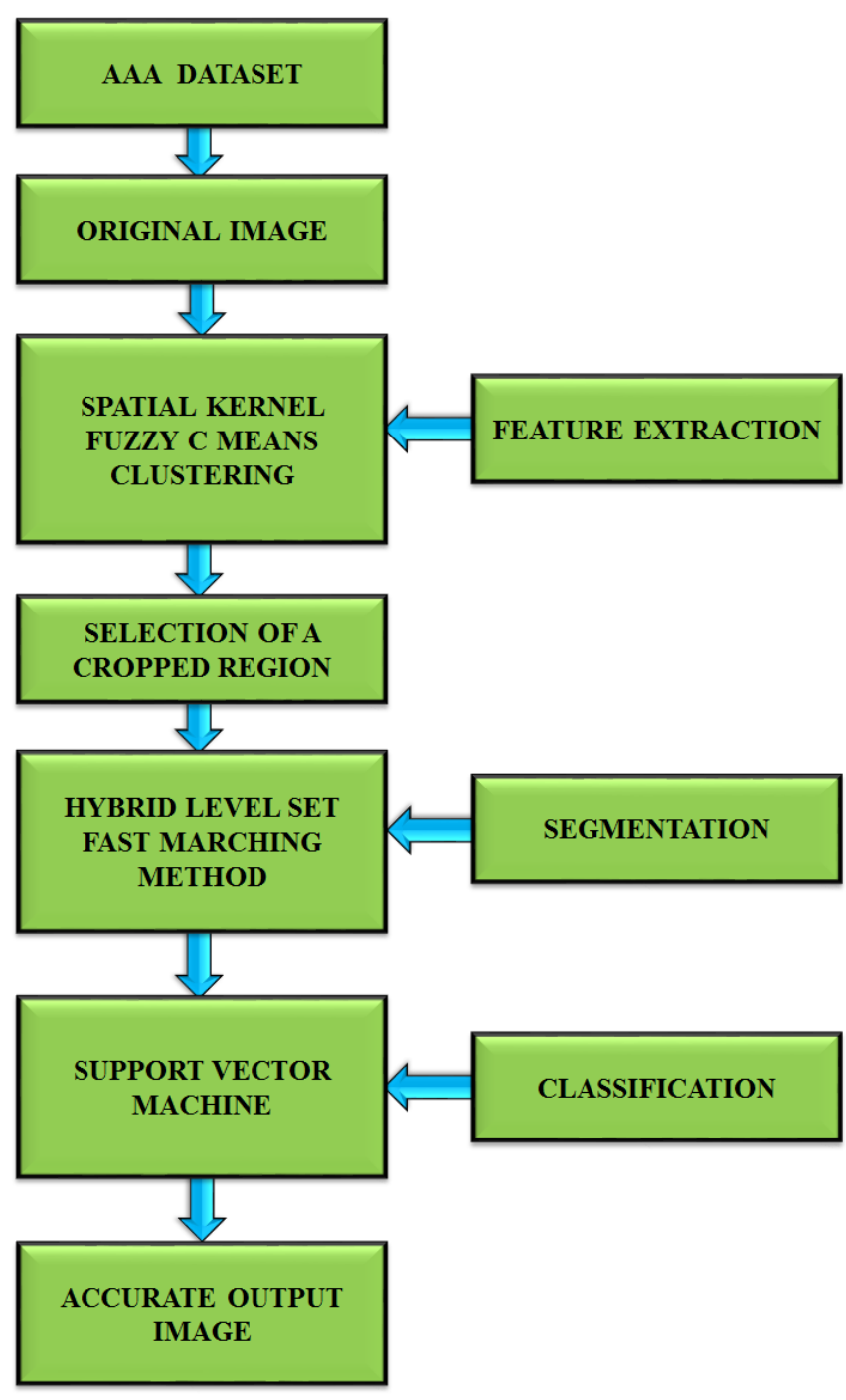

Fig 1: Process Flow of AAA identification

The proposed work shown in figure 1 is split into four levels as preprocessing, feature extraction, segmentation and classification.

\subsection{PRE-PROCESSING}

The obtained original RGB images from the dataset are transformed into Gray scale images using conventional color conversion method. The preprocessing complexity is thus minimized and the processing will be done quickly.

The image is split into a series of blocks with $\mathrm{N} \times \mathrm{N}$ (pixel) size for each block and this is the essential unit of the change detection. This method is known as "hot spot" identification.

\subsection{FEATURE EXTRACTION}

To simplify the resource quantities which are required for accurate elaboration of a large set of information, feature extraction is used. For feature extraction Spatial Kernel FCM transform is utilized. In numerical analysis and functional analysis, a Spatial Kernel FCM is used. The spatial kernel FCM allocates the chosen picture pixels in a database " $\mathrm{X}$ " and the centre values are assigned, finally membership esteems are tabulated.

In normal feature extraction process, a window from the picture with definite size is chosen. Then the phase concurrency amplitude and orientation for each pixel are calculated for the particular template to extricate the feature content of edge enhancement. Next, the template picture is divided into extend beyond block as spatial areas with cell having pixel. Finally, the extracted features for all blocks are collected over the template window. Thus the feature vectors are obtained and fed to the classifier.

\subsection{MEDIAN FILTER}

Median filter is utilized for denoising the obtained MRI picture. The image is divided into three domains such as spatial, frequency and wavelet domains. The two types of spatial domain filter are linear filter and non linear filter. Here we used a simple and powerful nonlinear median filter introduced by Tukeyet in 1977, familiar to eliminate salt and pepper noise (impulse). It is mostly utilized in the digital image processing to smoothen and filter for preserving edges in the images. Depending on the shape and size of the filter mask, the amount of noise is reduced. The intensity esteems among two points are also reduced by the median filter. In this filter mean value is placed instead of pixel value .Let the input image be $\mathrm{P}_{-}(\mathrm{x}, \mathrm{y})$

Steps in median filter are given below:

Select a 2-dimensional window $\mathrm{W}$ of size $3 \times 3$.

Compute V_med which is the median of the pixel esteems in windows is $\mathrm{V}$.

\section{P_(x,y) is replaced by V_med}

Repeat the steps until the total image is processed.

\subsection{SPATIAL KERNEL FCM (SKFCM)}

In image processing grouping and division are very closest terms. Pixels with similar attributes are split depending on the variables like spatial data and distance measures in the clustering methods. The result of the grouping process is delineated in the spatial domain as various areas for segmentation process. Several resemblance metric like interval, affinity, amplitude are divided in clustering. Spatial FCM (SFCM) was introduced in order to reduce the noises and outliers but less amount of noise is removed. Spatial Kernel Fuzzy C Means Clustering (SKFCM) is introduced to provide better and faster segmentation result compare to SFCM and FCM alone. Using conventional FCM method, SKFCM integrates spatial data and utilizes Gaussian RBF kernel operator. It gives best result in numerous applications.

\subsubsection{SKFCM ALGORITHM}

There are six steps:

$>$ Assign the chosen picture pixels of the database " $\mathrm{X}$ " and fix the centre value $\varepsilon$ with $m$ as weighting exponent for every fuzzy membership function.

$>$ Sponsorship values $\mathrm{U}_{-} \mathrm{ij}$ are computed against every centres of the pixels such that.

$U_{i j}=\frac{\left(1-K\left(x_{j}, v_{i}\right)\right)^{-1 /(m-1)}}{\sum_{k=1}^{c}\left(1-K\left(x_{j_{i}} v_{i}\right)\right)^{-1 /(m-1)}}$

$>$ calculate new centre values $V_{i}$

$V_{i}=\frac{\sum_{j=1}^{n} W_{i j}^{m} x_{j}}{\sum_{j=1}^{n} W_{i j}^{m}}$

$>W_{i j}$ is a new membership value which is calculated,

$W_{i j}=\frac{v_{i j}^{p} s_{i j}^{q}}{\sum_{k=1}^{c} U_{i j}^{p} j_{i j}^{q}}$

$S_{i j}=\sum_{k \in N K\left(x_{j}\right)} U_{i j}$

Here $\mathrm{NK}\left(x_{j}\right)$ represents a block window lied in the spatial domain $x_{j}$

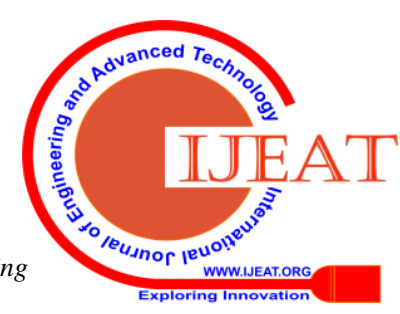


$>\mathrm{J}$ is Objective function which is determined by,

$J=2 \sum_{i=1}^{c} \sum_{j=1}^{N} W_{i j}^{m}\left(1-K\left(x_{j}, v_{i}\right)\right)$

$>$ The condition $\{\mathrm{J}(\mathrm{i})-\mathrm{J}(\mathrm{i}=1)\}<\varepsilon$ for threshold of termination is evaluated with termination condition as $\mathcal{E}$. Stop the procedure if the criteria is satisfied, otherwise repeat from the second step until it satisfies the condition.

\subsection{SEGMENTATION AND CLASSIFICATION}

The HLSFMM removes time-dependent velocity without any limit on the sign. The raw grey scale pictures are processed to enhance the structure visualization which is used in analysis. After the feature extraction, dimensionality minimization is performed on the feature vector and the segmented picture will be classified using a supervised machine learning algorithm (Support Vector Machine (SVM)). It can classify and regret the issues by observing the shape and size variations, of the image to produce the accurate results.

\subsubsection{HBLSFM method}

Active contour is denoted as Level Set Method (LSM). The level set function (LSF) $\phi(x, y, t)$ with outline as zero level set $\mathrm{X}(\mathrm{s}, \mathrm{t})$ is

$$
\phi[\mathrm{X}(\mathrm{s}, \mathrm{t}), \mathrm{t}]=0
$$

Dynamic parametric contour $\mathrm{X}(\mathrm{s}, \mathrm{t})$ created from ACM with $\mathrm{s}$ as parameter and $\mathrm{t}$ as time normal direction of the curve circulation is represented as,

$$
\frac{\partial x}{\partial t}=\mathrm{FN}
$$

Where speed is denoted by $F$ and normal vector is $\mathrm{N}$ for $\mathrm{X}$ curves. Equation (1) represents the curve evolution captivated by extra terms $\mathrm{X}(\mathrm{s}, \mathrm{t})$ as a time varying LSF $\phi(x, y, t)$. This assumes positive esteems outside the level set and negative esteems inside the level set, and the normal vector is given by, $N=(\nabla \phi|\nabla \phi|)$.

Equation (2) is rewritten by using equation (1)

$$
\frac{\partial \emptyset}{\partial t}=\mathrm{F}|\nabla \phi|
$$

where, $\nabla$ - gradient operator. Level set evolution (LSE) considers the benefit of expressing outlines in complex topology and represented in the Equation .3. By using regularity re-initialization techniques the drawbacks can be eliminated and defined mathematically as,

$$
\frac{\partial \emptyset}{\partial t}=\operatorname{sign}\left(\phi_{0}\right)(1-|\nabla \phi|)
$$

Where, $\operatorname{sign}\left(\phi_{0}\right)$ - sign function of $\operatorname{sign} \phi_{0}$. Reinitialization problem is reduced in hybrid level set method. To illustrate the required location of zero level set outlines it utilizes distance regularization and energy terms. Amplitude gradient of LSF at its lower positions with the necessary contour is strained by potential function. Let $\phi: \Omega \rightarrow R$ be the LSF in $\Omega$ domain and energy function is represented as

$$
\varepsilon(\phi)=\mu R_{p}^{(\phi)}+\varepsilon_{e x t}(\phi)
$$

$R_{p}^{(\phi)}$ - Regularization term. $\mu$ - Constant. $\varepsilon_{e x t}(\phi)$ - Energy external term. $R_{p}^{(\phi)}$ can be defined as,

$R_{p}^{(\phi)}=\varepsilon_{e x t}(\phi) \int_{\Omega} p(|\nabla \phi|) d x$

$\mathrm{P}$ - Energy density function. $\varepsilon_{e x t}$ - External energy, which is used to accomplish a minimum position during the zero level set of LSF $\phi$. The external energy can be minimized by solving LSE equations. The $p$ of regularized separation is represented as,

$$
p=p_{1}(s)=\frac{1}{2}(s-1)^{2}
$$

$p_{1}(s)$ - Potential function. Where $\mathrm{s}=1$ is the minimum potential value. $R_{p}^{(\phi)}$ is mathematically given by,

$$
p(\phi)=\frac{1}{2} \int_{\Omega}(|\nabla \phi-1|)^{2}
$$

From equation (6) $R_{p}^{(\phi)}$ Gateaux derivative function of which is given by,

$$
\frac{\partial R_{p}}{\partial \phi}=-\operatorname{div}\left(d_{p}(|\nabla \phi|) \nabla \phi\right)
$$

From linearity of Gateaux derivative and equation (5) is written as

$$
\begin{aligned}
& \frac{\partial \varepsilon}{\partial \phi}=\mu \frac{\partial R_{p}}{\partial \phi}+\frac{\partial \varepsilon_{e x t}}{\partial \phi} \\
& \frac{\partial \varepsilon}{\partial \phi} \text { - Gateaux derivation of outer ener } \\
& \text { (9) and (10), } \\
& \frac{\partial \varepsilon}{\partial \phi}=\mu \operatorname{div}\left(d_{p}(|\nabla \phi|) \nabla \phi\right)-\frac{\partial \varepsilon_{e x t}}{\partial \phi}
\end{aligned}
$$$$
\frac{\partial \varepsilon}{\partial \phi} \text { - Gateaux derivation of outer energy. From the equations }
$$

The influence of regularization separation in HLSFME can be seen from equations (10) and (11) from which the $\mu R_{p}(\phi)$ gradient of the energy given by,

$$
\frac{\partial \phi}{\partial t}=\mu \operatorname{div}\left(d_{p}(|\nabla \phi|) \nabla \phi\right)
$$

Let us consider $I_{0}$ as the image on $\Omega$ domain, edge indicator term is $\mathrm{g}$ which is defined as,

$$
g=\frac{1}{1+\left|\nabla G_{\pi}+I_{n}\right|}
$$

$G_{\sigma}$ - Gaussian kernel for image smoothening

.LSF $\phi: \Omega \rightarrow R$, energy function $\varepsilon(\phi)$ is given by,

$\varepsilon(\phi)=\mu R_{p}(\phi)+\lambda L_{g}(\phi)+\alpha A_{g}(\phi)$

where

$$
\varepsilon_{e x t}(\phi)=\lambda L_{g}(\phi)+\alpha A_{g}(\phi)
$$

$\lambda>0, \alpha \in R$ is the coefficient of energy function $L_{g}(\phi)$ and $A_{g}(\phi)$ is identified as

$$
\begin{aligned}
L_{g}(\phi)=\int_{\Omega} g \delta(\phi)|\nabla \phi| d x(15) \\
A_{g(\phi)}=\int_{\Omega} g H(-\phi) d x
\end{aligned}
$$

$\mathrm{H}$ - Heavy side functional, $\delta$ - Dirac delta functional, $L_{g}(\phi)$ - used to calculate the integration of g via zero LSE of $(\phi)$. $A_{g(\phi)}$ - calculates the weighted location of the region. $\delta$, $\mathrm{H}, L_{g}, A_{g}$ - estimated by smoothening functions $\delta_{\varepsilon}$ and $H_{\varepsilon}$. The energy function is given by,

$$
\begin{aligned}
& \varepsilon_{\varepsilon}(\phi)=\mu \int_{\Omega} p(|\nabla \phi|) d x+\lambda \int_{\Omega} g \delta_{\varepsilon}(\phi)|\nabla \phi| d x+ \\
& \alpha \int_{\Omega} g H_{\varepsilon}(-\phi) d x
\end{aligned}
$$

Solving gradient flow, the vitality function equation (17) is reduced by

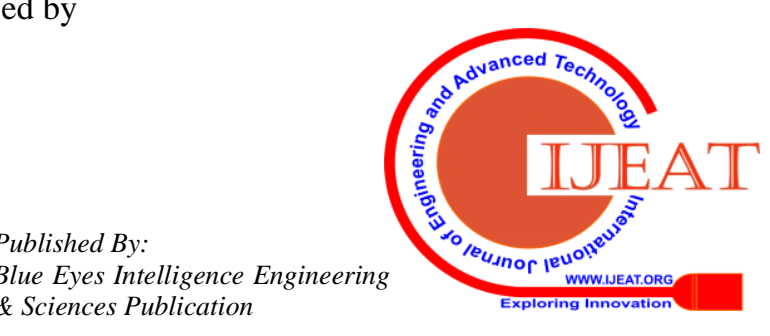


$\frac{\partial \phi}{\partial t}=\mu \operatorname{div}\left(d_{p}(|\nabla \phi|) \nabla \phi\right)+\lambda \delta_{\varepsilon}(\phi) \operatorname{div}\left(\frac{\nabla \phi}{|\nabla \phi|}\right)+\alpha g \delta_{\varepsilon}(\phi)$

First term is denoted as distance regularization. Balance two terms are denoted as energy .It is the edge based model used for segmentation.
Table shows the validation metrics for the experiments performed with conventional LSM and HLSFMM algorithm, With the HLSFM algorithm, the maximum Dice similarity coefficient is obtained by cluster is identified as a thrombus. Compared with the conventional level set method HLSFMM is efficient.

\section{EXPERIMENTAL RESULTS}

\begin{tabular}{|c|c|c|c|c|c|}
\hline Method & Overlap & Jaccard & Dice & FN & FP \\
\hline LSM 1 & $.80 \pm .06$ & $.69 \pm .10$ & $.81 \pm .07$ & $.20 \pm .06$ & $.18 \pm .10$ \\
\hline LSM 2 & $.79 \pm .08$ & $.69 \pm .11$ & $.81 \pm .08$ & $.21 \pm .08$ & $.15 \pm .11$ \\
\hline LSM 3 & $.77 \pm .07$ & $.70 \pm .11$ & $.80 \pm .08$ & $.21 \pm .07$ & $.12 \pm .11$ \\
\hline HLSFMM 1 & $.87 \pm .09$ & $.74 \pm .08$ & $.85 \pm .06$ & $.12 \pm .07$ & $.18 \pm .06$ \\
\hline HLSFMM 2 & $.78 \pm .09$ & $.69 \pm .11$ & $.81 \pm .09$ & $.23 \pm .90$ & $.15 \pm .10$ \\
\hline HKSFMM 3 & $.80 \pm .08$ & $.70 \pm .10$ & $.82 \pm .08$ & $.20 \pm .08$ & $.16 \pm .10$ \\
\hline
\end{tabular}

In this paper CT MRI of AAA image is taken. The original AAA MRI with noise is shown in figure 2. Median filter is utilized for denoising the image and the denoised picture is presented in figure 3 . This denoised picture is given to

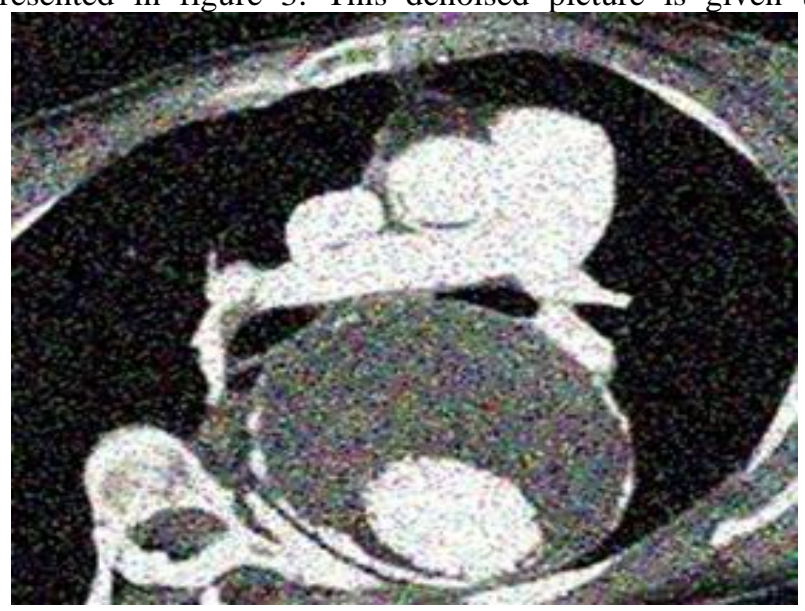

Fig2. Input image for Pre-processing

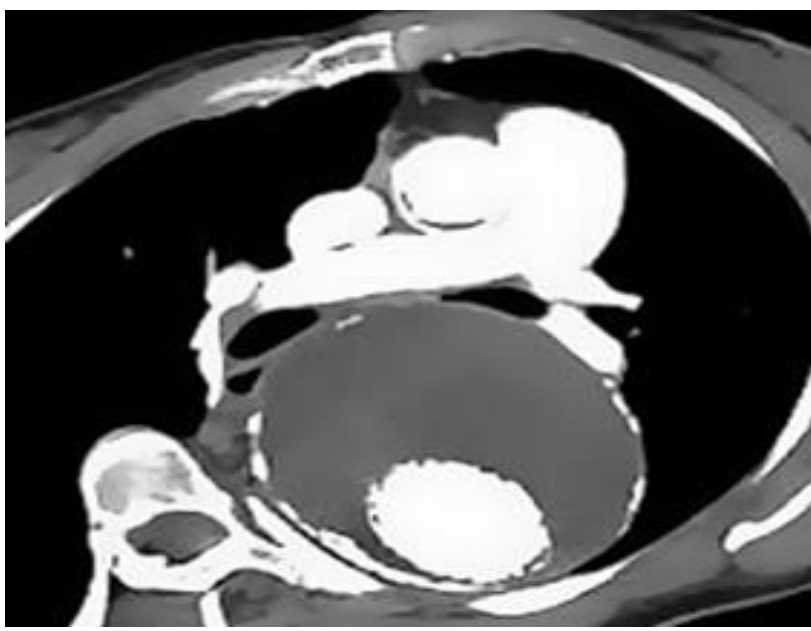

Fig3. Median filter output image

SKFCM algorithm for obtaining the C-means clustered image with enhanced edge as given in figure 3 . After preprocessing stage, HLSFMM is performed on the picture to obtain the final segmented picture (Fig 5).

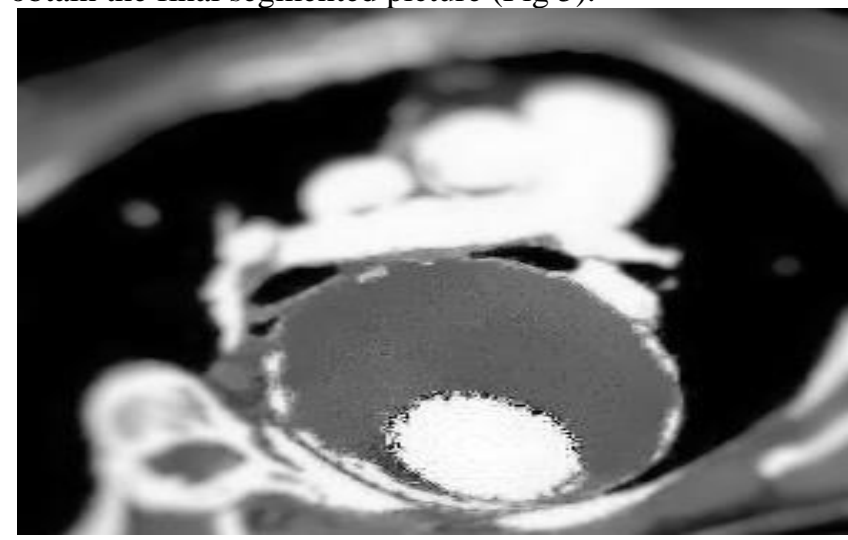

Fig4. Binary Converted Image

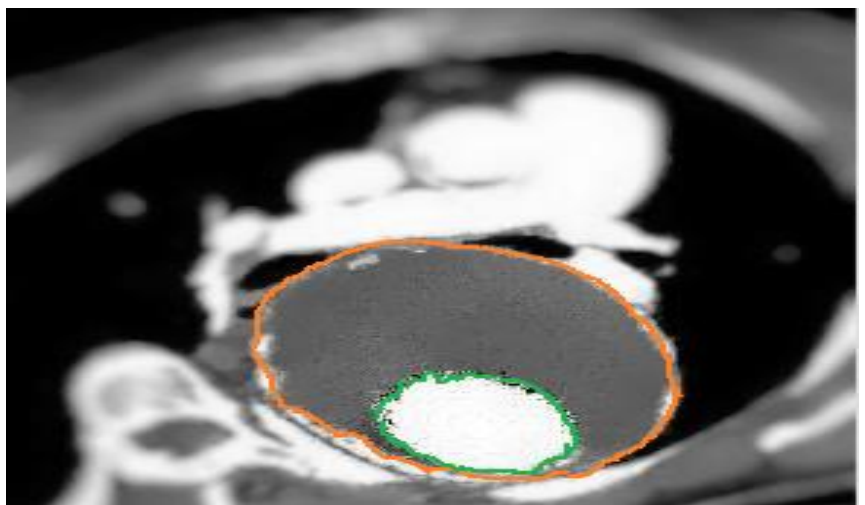

Fig5. Clustered Image with Fuzzy C means algorithm

Exploring Innovation




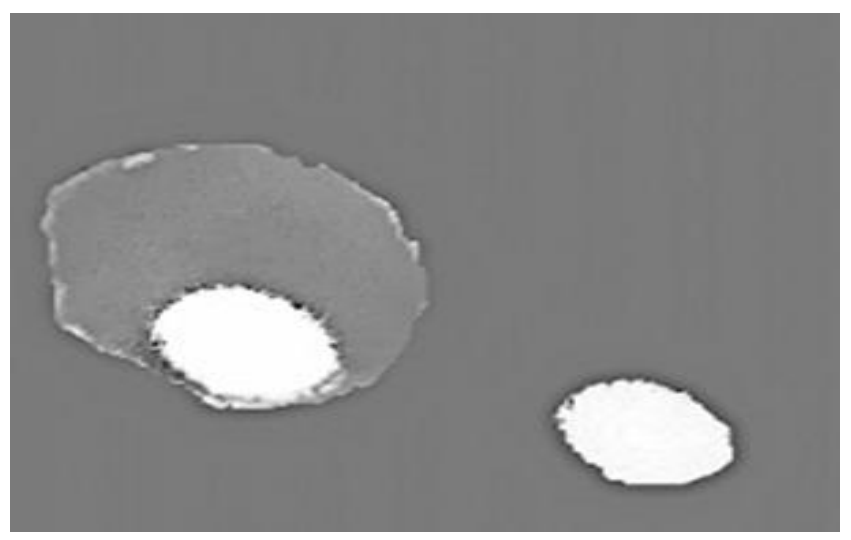

Fig6. Segmented Image using proposed approach

Table 1 and 2 Accuracy, precision, F-Score and computational time comparison table

\begin{tabular}{|c|c|c|c|c|}
\hline \multirow{2}{*}{ IMAGE NO. } & \multicolumn{2}{|c|}{ ACCURACY } & \multicolumn{2}{c|}{ PRECISION } \\
\cline { 2 - 5 } & $\begin{array}{c}\text { WITHOUT } \\
\text { OPTIMIZATION }\end{array}$ & $\begin{array}{c}\text { WITH } \\
\text { OPTIMIZATION }\end{array}$ & $\begin{array}{c}\text { WITHOUT } \\
\text { OPTIMIZATION }\end{array}$ & $\begin{array}{c}\text { WITH } \\
\text { OPTIMIZATION }\end{array}$ \\
\hline IMAGE 1 & $\mathbf{8 2 . 5}$ & $\mathbf{8 5 . 4}$ & $\mathbf{8 2 . 6}$ & $\mathbf{8 5 . 8}$ \\
\hline IMAGE 2 & $\mathbf{8 1 . 2}$ & $\mathbf{8 6 . 7}$ & $\mathbf{8 3 . 8}$ & $\mathbf{8 7 . 2}$ \\
\hline IMAGE 3 & $\mathbf{8 3 . 7}$ & $\mathbf{8 6 . 9}$ & $\mathbf{8 4 . 2}$ & $\mathbf{8 7 . 6}$ \\
\hline IMAGE 4 & $\mathbf{8 2 . 9}$ & $\mathbf{8 6 . 4}$ & $\mathbf{8 5 . 6}$ & $\mathbf{8 8 . 1 5}$ \\
\hline IMAGE 5 & $\mathbf{8 3 . 3}$ & $\mathbf{8 8 . 3}$ & $\mathbf{8 4 . 7}$ & $\mathbf{8 7 . 4 9}$ \\
\hline
\end{tabular}

The above table provides the deviation MRI AAA images with HLSFMM segmentation and SVM classifier by conducting the experiment with the similar pictures.

\begin{tabular}{|c|c|c|c|c|}
\hline \multirow{2}{*}{ IMAGE NO. } & \multicolumn{2}{|c|}{ F- SCORE } & \multicolumn{2}{c|}{ COMPUTATION TIME(ns) } \\
\cline { 2 - 5 } & $\begin{array}{c}\text { WITHOUT } \\
\text { OPTIMIZATION }\end{array}$ & $\begin{array}{c}\text { WITH } \\
\text { OPTIMIZATION }\end{array}$ & $\begin{array}{c}\text { WITHOUT } \\
\text { OPTIMIZATION }\end{array}$ & $\begin{array}{c}\text { WITH } \\
\text { OPTIMIZATION }\end{array}$ \\
\hline IMAGE 1 & $\mathbf{8 0 . 9}$ & 75.5 & $\mathbf{0 . 8 2}$ & $\mathbf{0 . 9 3}$ \\
\hline IMAGE 2 & 78.5 & 76.8 & $\mathbf{0 . 8 4}$ & 0.96 \\
\hline IMAGE 3 & 76.4 & 74.2 & $\mathbf{0 . 8 0}$ & 0.84 \\
\hline IMAGE 4 & 74.6 & 75.6 & $\mathbf{0 . 8 2}$ & $\mathbf{0 . 8 8}$ \\
\hline IMAGE 5 & $\mathbf{8 2 . 8}$ & 77.5 & $\mathbf{0 . 7 4}$ & $\mathbf{0 . 8 0}$ \\
\hline
\end{tabular}

The streamlining to decrease the hunt dimensionality would marginally expand the calculation extend however the execution of the proposed framework is well-improved with respect to order exactness, accuracy and f-score esteem. Time parameter is undermined with division and arrangement exactness.

\section{CONCLUSION}

A hybrid level set method is proposed in this research to detect and segment the picture taken from MRI of Abdominal aorta region. In standard level set method, reinitialization problems are high. Re-initialization problem is totally eliminated in the Hybrid Level Set Fast Marching method (HLSFMM). Median filter reduces the noise in the image efficiently. Associated with standard Fuzzy C Means algorithm, Support Vector Machine (SVM) is utilizing the Gaussian RBF kernel utility as a distance parameter by incorporating spatial data. This method is contrasted with the recent extraction methods. The performance is evaluated in terms of Accuracy, coefficient, overall presentation and error rate and noise.

\section{REFERENCES}

1. P. Polterauer, T. Hlzenbein, J. Nanobashvilli, et al., .Das abdominal aortic aneurysm,. Wiener Medizinische Wochenschrift 6a, pp. 15.18, 1998.

2. Physiology and function from multidimensional images,. in Proceedings of the SPIE Medical Imaging, E. A. Hoffman, ed., pp. 323.337, 1996

3. A. P. Dhawan and S. Juvvadi, .Knowledge-based analysis and understanding of medical images, Computer Methods and Programs in Biomedicine 33, pp. 221 .239, 1990.

4. M. Garreau, J. L. Coatrieux, R. Collorec, and C. Chardenon, A knowledge-based approach for 3-d reconstruction and labeling of vascular networks from biplane angiographic projections,. IEEE Transactions on Medical Imaging 10, pp. 122 .131, 1991.

5. S. Loncaric, D. Kovacevic, and E. Sorantin, .Semi-automatic active contour approach to segmentation of computed tomography volumes, in Proceedings of SPIE Medical Imaging, 3979, 2000.

6. Sakalihasan N, Limet R, Defawe O D., Abdominal Aortic Aneurysm. Lancet 2005; 365: 1577-89

7. Blankensteijn J D., Impact of the EVAR-1 and DREAM Trials, Endovascular Today March 2005 
8. Blankensteijn J D, de Jong S E C A, Prinssen M et al M. Verhagen, E. Buskens and D. E. Grobbee .,Two-Year Outcomes after Conventional or Endovascular Repair of Abdominal Aortic Aneurysms, N Engl J Med, 352:2398,2005.

9. Bodur O, Grady L, Stillman A et al.,Semi-automatic aortic aneurysm analysis. Proceedings of the SPIE, Volume 6511, pp.65111, 2007.

10. Greiner K, Egger J, Grobkopf S et al ., Segmentation of aortic aneurysms in CTA-images with the statistic method of the Active Appearance Models (in German). Proceedings of Bildverarbeitung für die Medizin (BVM), Berlin, Germany, Springer-Verlag, Apr. 2008

11. Lu J, Egger J, Wimmer A et al ., Segmentation and visualization of lumen und thrombus of thoracic aortic aneurysms (in German). Proceedings of 6. Jahrestagung der Deutschen Gesellschaft für Computer-und Roboter assistierte Chirurgie (CURAC), Karlsruhe, Germany, pp. 251-254, Oct. 2007

12. De Bruijne M, van Ginneken B, Niessen W J et al ., Adapting active shape models for 3D segmentation of tubular structures in medical images. Information Processing in Medical Imaging, Ambleside, UK, Vol. 2732, pp. 136-147,2003

13. Heimann T. Et al., "New methods for leak detection and contour correction in seeded region growing segmentation," Int. Archives of Photogrammetry and Remote sensing, vol.35, pp. 16, 2004.

14. B.H.Sollie: Automatic segmentation and registration of CT and US images of abdominal aortic aneurysm using ITK (2002) Masterthesis: Norwegian University of Science and Technology, Norway.

15. T.Behrens, K.Rohr, H.S.Stiehl: Robust segmentation of tubular structures in 3-D medical images by parametric object detection and traking. IEEE Trans Syst Man Cybern B 33 (2003) 554-561

16. S. Habib, J. Dehmeshki, "Automatic segmentation of Abdominal Aortic

Aneurysm”, IEEE transactions on CSIT .September, 2018.

17. B. B. Nakhjavanlo1, T. J. Ellis2, P.H.Soan3, J. Dehmeshki4 "3D Medical Image Segmentation Using Level Set Models and Anisotropic Diffusion" computer society .2011

18. D. Magee A. Bulpitt E. Berry "3D Automated Segmentation and Structural Analysis of Vascular Trees Using Deformable Models" IEEE conference on computer vision. July, 2001.

19. R.G.P. Lopata1, S.P.L. Meesters1, V.L. Nguyen2, G.W.H. Schurink2 and F.N. van de Vosse 1"Automated 2D Ultrasound Fusion Imaging of Abdominal Aortic Aneurysms" IEEE international ultrasonic's symposium. Oct, 2012.

20. M. Auer and T. Christian Gasser* "Reconstruction and Finite Element Mesh Generation of Abdominal Aortic Aneurysms from Computerized Tomography Angiography Data with Minimal User Interactions" IEEE Transactions on Medical Imaging, Vol. 29, NO. 4, April 2010.

21. Moti Freiman, Steven J. Esses, Leo Joskowicz, Jacob Sosna, "An Iterative Model Constrained Graph-Cut Algorithm For Abdominal Aortic Aneurysm Thrombus Segmentation", IEEE Transactions 2010.

22. Roger C.Tam, Christopher G.Healey, Borys Flak and peter Cahoon, "Volume Rendering of Abdominal Aortic Aneurysms", IEEE Visualization '97, March 31, 1997

23. Achia Kronman, Leo Joskowicz, Jacob Sosna ,"Automatic Detection And Correction Of Segmentation Leaks In Medical Images", 2010.

24. J. Egger , T. O’Donnell , C. Hopfgartner and B. Freisleben, "Graph-based Tracking Method for Aortic Thrombus Segmentation", Springer, IFMBE Proceedings 22, pp. 584-587, r 2009

25. Josu Maiora and Manuel Grana, "A Hybrid Segmentation of Abdominal CT Images”, Springer, LNCS 7209, pp. 416-423, 2012.

26. Stefanie Demirci, Guy Lejeune, Nassir Navab "Hybrid Deformable Model for Aneurysm Segmentation" IEEE international conference on biomedical imaging. Aug, 2009.

27. Tuan D. Pham1, Jonathan Golledge2 "Pattern Analysis of Imaging Markers in Abdominal Aortic Aneurysms" IEEE international conference on bio medical engineering and informatics. Dec, 2013.

28. B. B. Nakhjavanlo1, T. J. Ellis2, P.H.Soan3, J. Dehmeshki4 "3D Medical Image Segmentation Using Level Set Models and Anisotropic Diffusion" International conference on signal image technology and internet based system. Dec, 2011.

29. Cosmin Adrian Morariu, Malte Thomas, Josef Pauli, Daniel Sebastian Dohle, Konstantinos Tsagakis "Sequential vs. Batch Machine Learning with

Evolutionary Hyper parameter Optimization for Segmenting Aortic Dissection Thrombus" International conference on pattern recognition. Dec, 2016

30. Marleen de Bruijne, Wiro J. Niessen, Associate Member, J. B. Antoine Maintz, and Max A. Viergever, Member "Localization and Segmentation of Aortic Endografts Using Marker Detection" IEEE Transactions On Medical Imaging . Volume: 22, Issue: 4 , April 2003.

\section{AUTHORS PROFILE}

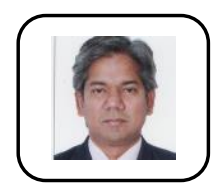

S. Anandh is a research scholar in the Department of Biomedical Engineering, Bharath University, Chennai. He has teaching experience of 15 years. His area of interest are Medical Image processing, Medical Signal processing and biomedical Instrumentation.

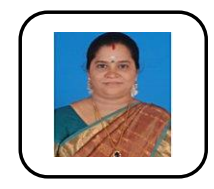

Dr. R. Vasuki is working as a Professor and Head in the Department of Biomedical Engineering, BharathUniversity, Chennai. She has $20+$ years of teaching experience.Her areas of interest are Bioinformatics, Bio-signal processing and Image processing

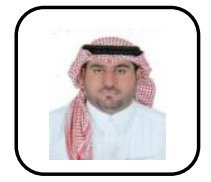

Dr. Raid Al Baradie is working as an Associate Professor in the Department of Medical Lab, Majmaah University, Kingdom of Saudi Arabia. He has received Doctorate in the Faculty of Medicine and Human Sciences, Manchester University, United Kingdom. His areas of interest are human Anatomy and Physiology, Pathology and Histology. 